\title{
On the Existence of Variational Principles for a Class of the Evolutionary Differential-Difference Equations
}

\author{
I. A. Kolesnikova and V. M. Savchin \\ University of Russia, Miklukho-Maklaya Street 6, Moscow 117198, Russia \\ Correspondence should be addressed to V. M. Savchin, vsavchin@yandex.ru \\ Received 10 March 2011; Accepted 18 October 2011 \\ Academic Editor: V. Stepanov
}

Copyright (C) 2012 I. A. Kolesnikova and V. M. Savchin. This is an open access article distributed under the Creative Commons Attribution License, which permits unrestricted use, distribution, and reproduction in any medium, provided the original work is properly cited.

Necessary and sufficient conditions for the existence of variational principles for a given wide class of evolutionary differential-difference operator equation are obtained. The theoretic results are illustrated by two examples.

\section{Introduction.}

We consider the equation:

$$
N(u)=0, \quad u \in D(N)
$$

where $D(N)$ is a domain of the definition of the operator $N: D(N) \subseteq U \rightarrow V$, and $U, V$ are normed linear spaces over the field of real numbers $\mathbb{R}$.

Later, we will assume that at every point $u \in D(N)$, there exists the Gâteaux derivative $N_{u}^{\prime}$ of $N$ defined by the formula $\left.(d / d \epsilon) N(u+\epsilon h)\right|_{\epsilon=0}=\delta N(u, h)=N_{u}^{\prime} h$.

The operator $N: D(N) \rightarrow V$ is said to be potential [1] on the set $D(N)$ relative to a given bilinear form $\Phi(\cdot, \cdot): V \times U \rightarrow R$, if there exists a functional $F_{N}: D\left(F_{N}\right)=D(N) \rightarrow R$ such that

$$
\delta F_{N}[u, h]=\Phi(N(u), h), \quad \forall u \in D(N), \forall h \in D\left(N_{u}^{\prime}\right)
$$

In that case, we also say that the given equation admits the direct variational formulation. 
A problem of the construction of the functional $F_{N}$ upon the given operator $N$ is known as the classical inverse problem of the calculus of variations [1]. Note that practically no one has been solving inverse problem of the calculus of variations for partial differential-difference operators until recently [2-4]. Let us also note that for a wide classes of partial differential equations, there has been developped the problem of recongnition of variationality upon the structure of corresponding operators $[5,6]$. There is a theoretical and practical interst in the extention of these results on partial differential-difference equations [7-9].

For what follows, we suppose that $D(N)$ is a convex set, and we also need the following potentiality criterion [1]:

$$
\Phi\left(N_{u}^{\prime} h, g\right)=\Phi\left(N_{u}^{\prime} g, h\right), \quad \forall u \in D(N), \forall h, g \in D\left(N_{u}^{\prime}\right)
$$

Under this condition, the potential $F_{N}$ is given by

$$
F_{N}[u]=\int_{0}^{1} \Phi\left(N\left(u_{0}+\lambda\left(u-u_{0}\right)\right), u-u_{0}\right) d \lambda+\text { const, }
$$

where $u_{0}$ is a fixed element of $D(N)$.

The functional $F_{N}$ is called the potential of the operator $N$, and in turn the operator $N$ is called the gradient of the functional $F_{N}$.

\section{Statement of the Problem.}

Let us consider the following differential-difference operator equation:

$$
N(u) \equiv \sum_{\lambda=-1}^{1} P_{\lambda}(t) u_{t}(t+\lambda \tau)+Q(t, u(t+\lambda \tau))=0, \quad u \in D(N), t \in\left[t_{0}, t_{1}\right] \subset \mathbb{R}
$$

Here, $P_{\lambda}: U_{1} \rightarrow V_{1}(\lambda=-1,0,1)$ are linear operators depending on $t ; Q:\left[t_{0}-\tau, t_{1}+\right.$ $\tau] \times U_{1}{ }^{3} \rightarrow V_{1}$ is in general a nonlinear operator; $N: D(N) \subseteq U \rightarrow V ; U=C^{1}\left(\left[t_{0}-\tau, t_{1}+\right.\right.$ $\left.\tau] ; U_{1}\right) ; V=C\left(\left[t_{0}-\tau, t_{1}+\tau\right] ; V_{1}\right)$, where $U_{1}, V_{1}$ are real normed linear spaces, $U_{1} \subseteq V_{1}$.

The domain of definition $D(N)$ is given by the equality:

$$
D(N)=\left\{u \in U: u(t)=\varphi_{1}(t), t \in\left[t_{0}-\tau, t_{0}\right], u(t)=\varphi_{2}(t), t \in\left[t_{1}, t_{1}+\tau\right]\right\}
$$

where $\varphi_{i},(i=1,2)$ are given functions.

Under the solution of (2.1), we mean a function $u \in D(N)$ satisfying the identity:

$$
N(u) \equiv \sum_{\lambda=-1}^{1} P_{\lambda}(t) u_{t}(t+\lambda \tau)+Q(t, u(t+\lambda \tau))=0, \quad \forall t \in\left[t_{0}, t_{1}\right] \subset \mathbb{R}
$$


Journal of Function Spaces and Applications

Let us give the following bilinear form:

$$
\Phi(\cdot, \cdot) \equiv \int_{t_{0}}^{t_{1}}\langle\cdot, \cdot\rangle d t: V \times U \longrightarrow \mathbb{R}
$$

where the bilinear mapping $\Phi_{1} \equiv\langle\cdot, \cdot\rangle$ satisfies the following conditions:

$$
\begin{gathered}
\left\langle v_{1}, v_{2}\right\rangle=\left\langle v_{2}, v_{1}\right\rangle, \quad \forall v_{1}, v_{2} \in V_{1}, \\
D_{t}\langle v, g\rangle=\left\langle D_{t} v, g\right\rangle+\left\langle v, D_{t} g\right\rangle, \quad \forall v, g \in V .
\end{gathered}
$$

Our aim is to define the structure of operators $P_{\lambda}(\lambda=-1,0,1)$ and $Q$ under which (2.1) allows the solution of the inverse problem of the calculus of variations relative to the bilinear form (2.4) such that $D_{t}=d / d t$ is the antisymmetric operator on $D\left(N_{u}^{\prime}\right)$, that is,

$$
\Phi\left(D_{t} h_{1}, h_{2}\right)=-\Phi\left(D_{t} h_{2}, h_{1}\right), \quad \forall h_{1}, h_{2} \in D\left(N_{u}^{\prime}\right)
$$

\section{Conditions of Potentiality and the Structure of (2.1) in the Case of Its Variationality.}

We denote by $K^{*}$ the operator adjoint to $K$.

Theorem 3.1. If $D_{t}^{*}=-D_{t}$ on the set $D\left(N_{u}^{\prime}\right)$, then for the existence of the direct variational formulation for the operator (2.1) on the set $D(N)$ relative to (2.4), it is necessary and sufficient that the following conditions hold on the set $D\left(N_{u}^{\prime}\right)$ :

$$
\begin{array}{r}
P_{-\lambda}+\left.P_{\lambda}^{*}\right|_{t \rightarrow t-\lambda \tau}=0,\left.\quad \frac{\partial P_{\lambda}^{*}}{\partial t}\right|_{t \rightarrow t-\lambda \tau}+Q_{u(t-\lambda \tau)}^{\prime}-\left.Q_{u(t+\lambda \tau)}^{\prime}{ }^{*}\right|_{t \rightarrow t-\lambda \tau}=0, \\
\lambda=-1,0,1, \quad \forall u \in D(N), \quad \forall t \in\left(t_{0}, t_{1}\right) .
\end{array}
$$

Proof. Taking into account formula (2.1), we get

$$
N_{u}^{\prime} h=\sum_{\lambda=-1}^{1} P_{\lambda} h_{t}(t+\lambda \tau)+\sum_{\lambda=-1}^{1} Q_{u(t+\lambda \tau)}^{\prime} h(t+\lambda \tau)
$$

The criterion of potentiality takes the following form:

$$
\begin{aligned}
& \int_{t_{0}}^{t_{1}}\langle\left.\sum_{\lambda=-1}^{1}\left\{P_{\lambda} h_{t}(t+\lambda \tau)+Q_{u(t+\lambda \tau)}^{\prime} h(t+\lambda \tau)\right\}, g(t)\right\rangle d t \\
& \quad=\int_{t_{0}}^{t_{1}}\left\langle\sum_{\lambda=-1}^{1}\left\{P_{\lambda} g_{t}(t+\lambda \tau)+Q_{u(t+\lambda \tau)}^{\prime} g(t+\lambda \tau)\right\}, h(t)\right\rangle d t,
\end{aligned}
$$


or

$$
\begin{aligned}
\int_{t_{0}}^{t_{1}} & {\left[\left\langle\sum_{\lambda=-1}^{1}\left\{P_{\lambda} h_{t}(t+\lambda \tau)+Q_{u(t+\lambda \tau)}^{\prime} h(t+\lambda \tau)\right\}, g(t)\right\rangle\right.} \\
& \left.-\left\langle\sum_{\lambda=-1}^{1}\left(P_{\curlywedge} g_{t}(t+\lambda \tau)+Q_{u(t+\lambda \tau)}^{\prime} g(t+\lambda \tau)\right), h(t)\right\rangle\right] d t=0, \quad \forall u \in D(N), \forall g, h \in D\left(N_{u}^{\prime}\right) .
\end{aligned}
$$

Bearing into account the condition $D_{t}^{*}=-D_{t}$ on the set $D\left(N_{u}^{\prime}\right)$, from (3.4), we get

$$
\int_{t_{0}}^{t_{1}}\left[\left\langle\sum_{\lambda=-1}^{1}\left(P_{\lambda} D_{t}+Q_{u(t+\lambda \tau)}^{\prime}\right) h(t+\lambda \tau), g(t)\right\rangle-\sum_{\lambda=-1}^{1}\left\langle\left[-D_{t}\left(P_{\lambda}^{*} h(t)\right)+Q_{u(t+\lambda \tau)}^{\prime}{ }^{*} h(t)\right], g(t+\lambda \tau)\right\rangle\right] d t=0
$$

or

$$
\begin{aligned}
\int_{t_{0}}^{t_{1}} & {\left[\left\langle\sum_{\lambda=-1}^{1}\left(P_{-\lambda} D_{t}+Q_{u(t-\lambda \tau)}^{\prime}\right) h(t-\lambda \tau), g(t)\right\rangle\right.} \\
& \left.-\left\langle\sum_{\lambda=-1}^{1}-\frac{\partial P_{\lambda}^{*}}{\partial t}-P_{\lambda}^{*} D_{t}+\left.Q_{u}^{\prime *}\right|_{t \rightarrow t-\lambda \tau} h(t-\lambda \tau), g(t)\right\rangle\right] d t=0 .
\end{aligned}
$$

Thus, condition (3.4) can be reduced to the following form:

$$
\begin{array}{r}
\int_{t_{0}}^{t_{1}} \sum_{\lambda=-1}^{1}\left\langle\left(P_{-\lambda} D_{t}+Q_{u(t-\lambda \tau)}^{\prime}\right) h(t-\lambda \tau)-\left.\left(-\frac{\partial P_{\lambda}^{*}}{\partial t}-P_{\lambda}^{*} D_{t}+Q_{u}^{\prime *}\right)\right|_{t \rightarrow t-\lambda \tau} h(t-\lambda \tau), g(t)\right\rangle d t=0, \\
\forall u \in D(N), \quad \forall g, h \in D\left(N_{u}^{\prime}\right) .
\end{array}
$$

This equality is fulfilled identically if and only if

$$
\sum_{\lambda=-1}^{1}\left[\left(P_{-\lambda}+\left.P_{\lambda}^{*}\right|_{t \rightarrow t-\lambda \tau}\right) D_{t}+\left.\frac{\partial P_{\lambda}^{*}}{\partial t}\right|_{t \rightarrow t-\lambda \tau}+Q_{u(t-\lambda \tau)}^{\prime}-\left.Q_{u(t+\lambda \tau)}^{\prime}{ }^{*}\right|_{t \rightarrow t-\lambda \tau}\right] \cdot h(t-\lambda \tau)=0,
$$

for all $u \in D(N)$. Thus, it is necessary and sufficient that conditions (3.1) hold. 
Journal of Function Spaces and Applications

Theorem 3.2. Conditions (3.1) are held if and only if (2.1) has the following form:

$$
\begin{array}{r}
N_{1}(u) \equiv \sum_{\lambda=-1}^{1}\left(\left.R_{\lambda}^{*}\right|_{t \rightarrow t-\lambda \tau}-R_{-\lambda}\right) u_{t}(t-\lambda \tau)+\left(\operatorname{grad}_{\Phi} B[u]-\sum_{\lambda=-1}^{1} \frac{\partial R_{\lambda}}{\partial t u}(t+\lambda \tau)\right)=0, \\
\forall u \in D\left(N_{1}\right), \quad t \in\left[t_{0}, t_{1}\right] \subset \mathbb{R} .
\end{array}
$$

The operators $R_{\lambda}$ and $B$ depend on $P_{\lambda}(t)$ and $Q(t, u(t+\lambda \tau))$.

Proof. If $D_{t}^{*}=-D_{t}$ on the set $D\left(N_{1 u}^{\prime}\right)$ and conditions (3.1) are held, then according to Theorem 3.1, operator (2.1) is potential on the set $D(N)$ relative to a given bilinear form (2.4).

Let us consider the following functional:

$$
F_{N}[u]=\int_{t_{0}}^{t_{1}}\left[\left\langle\sum_{\lambda=-1}^{1} R_{\lambda}(t) u(t+\lambda \tau), u_{t}(t)\right\rangle+B[u]\right] d t+F_{N}\left[u_{0}\right]
$$

It is easy to check that

$$
\begin{aligned}
\delta F_{N}[u, h]= & \int_{t_{0}}^{t_{1}}\left\langle\sum_{\lambda=-1}^{1} R_{\curlywedge} h(t+\lambda \tau), u_{t}(t)\right\rangle+\left\langle\sum_{\lambda=-1}^{1} R_{\curlywedge} u(t+\lambda \tau), h_{t}(t)\right\rangle+\left\langle\operatorname{grad}_{\Phi} B[u], h(t)\right\rangle d t \\
= & \int_{t_{0}}^{t_{1}}\left\langle\left.\sum_{\lambda=-1}^{1} R_{\lambda}^{*}\right|_{t \rightarrow t-\lambda \tau} u_{t}(t-\lambda \tau), h(t)\right\rangle-\left\langle\sum_{\lambda=-1}^{1} D_{t}\left(R_{\curlywedge} u(t+\lambda \tau)\right), h(t)\right\rangle \\
& +\left\langle\operatorname{grad}_{\Phi} B[u], h(t)\right\rangle d t \\
= & \int_{t_{0}}^{t_{1}}\left\langle\left.\sum_{\lambda=-1}^{1} R_{\lambda}^{*}\right|_{t \rightarrow t-\lambda \tau} u_{t}(t-\lambda \tau), h(t)\right\rangle-\left\langle\sum_{\lambda=-1}^{1} \frac{\partial R_{\lambda}}{\partial t} u(t+\lambda \tau), h(t)\right\rangle \\
& -\left\langle\sum_{\lambda=-1}^{1} R_{-\lambda} u_{t}(t-\lambda \tau), h(t)\right\rangle+\left\langle\operatorname{grad}_{\Phi} B[u], h\right\rangle d t \\
= & \int_{t_{0}}^{t_{1}}\left\langle\sum_{\lambda=-1}^{1}\left(\left.R_{\lambda}^{*}\right|_{t \rightarrow t-\lambda \tau}-R_{-\lambda}\right) u_{t}(t-\lambda \tau)\right. \\
& \left.+\left(\operatorname{grad}_{\Phi} B[u]-\sum_{\lambda=-1}^{1} \frac{\partial R_{\lambda}}{\partial t} u(t+\lambda \tau)\right), h(t)\right\rangle d t \\
= & \int_{t_{0}}^{t_{1}}\langle N(u), h\rangle d t, \quad \forall u \in D(N), \forall h \in D\left(N_{u}^{\prime}\right) .
\end{aligned}
$$

Then functional (3.10) is a potential of evolutionary operator (2.1). 
If $D_{t}^{*}=-D_{t}$ on $D\left(N_{u}^{\prime}\right)$, then

$$
\begin{gathered}
P_{-\lambda}(t)=\left.R_{\lambda}^{*}\right|_{t \rightarrow t-\lambda \tau}-R_{-\lambda,} \\
Q(t, u(t+\lambda \tau))=-\sum_{\lambda=-1}^{1} \frac{\partial R_{\lambda}}{\partial t} u(t+\lambda \tau)+\operatorname{grad}_{\Phi} B[u] .
\end{gathered}
$$

This theorem shows the structure of the given kind of differential-difference operator, which admits the solution of the inverse problem of the calculus of variations.

\section{Examples}

Example 4.1. Let us consider the evolutionary differential-difference equation with partial derivatives in the following form:

$$
N_{1}(u) \equiv \sum_{\lambda=-1}^{1}\left(a_{\lambda}(x, t) \frac{\partial u}{\partial t}(x, t+\lambda \tau)-b_{\lambda}^{i j}(x, t) \frac{\partial^{2} u}{\partial x_{i} \partial x_{j}}(x, t+\lambda \tau)\right)=0
$$

where $(x, t) \in Q=\Omega \times\left(t_{0}, t_{1}\right), t_{1}-t_{0}>2 \tau, i, j=\overline{1, n}, a_{\lambda}(x, t) \in C_{x, t}^{0,1}(\bar{Q}), b_{\lambda}^{i j}(x, t) \in C_{x, t}^{2,0}(\bar{Q})$.

$\Omega$ is a bounded domain in $\mathbb{R}^{n}$ with piecewise smooth boundary $\partial \Omega$.

The domain of definition $D\left(N_{1}\right)$ is given by the equality:

$$
\begin{gathered}
D\left(N_{1}\right)=\left\{u \in U=C_{x, t}^{2,1}\left(\bar{\Omega} \times\left[t_{0}-\tau, t_{1}+\tau\right]\right): u(x, t)=\varphi_{1}(x, t),(x, t) \in E_{1}=\bar{\Omega} \times\left[t_{0}-\tau, t_{0}\right],\right. \\
\left.u(x, t)=\varphi_{2}(x, t),(x, t) \in E_{2}=\bar{\Omega} \times\left[t_{1}, t_{1}+\tau\right],\left.\frac{\partial^{\mu} u}{\partial n_{x}^{\mu}}\right|_{\partial \Gamma_{\tau}}=\psi_{\mu}(x, t), \mu=0,1\right\},
\end{gathered}
$$

where $\Gamma_{\tau}=\partial \Omega \times\left[t_{0}-\tau, t_{1}+\tau\right], \varphi_{i} \in C\left(E_{i}\right),(i=0,1), \psi_{\mu} \in C\left(\Gamma_{\tau}\right),(\mu=0,1)$ are given functions. form (2.4).

We investigate the existence of variational principle for (4.1) relative to a given bilinear

For (4.1), we get

$$
N_{1 u}^{\prime} h=\sum_{\lambda=-1}^{1}\left(a_{\lambda}(x, t) h_{t}(x, t+\lambda \tau)-b_{\lambda}^{i j}(x, t) h_{x_{i} x_{j}}(x, t+\lambda \tau)\right)
$$


Journal of Function Spaces and Applications

Necessary and sufficient conditions of potentiality take the form:

$$
\begin{gathered}
\Phi\left(N_{1 u}^{\prime} h, g\right)-\Phi\left(N_{1 u}^{\prime} g, h\right) \\
\equiv-\int_{t_{0}}^{t_{1}} \int_{\Omega}\left\{\left(\left.\sum_{\lambda=-1}^{1} D_{t}\left(a_{\lambda}(x, t) g(x, t)\right)\right|_{t \rightarrow t-\lambda \tau}\right.\right. \\
\left.+\left.\left(\sum_{\lambda=-1}^{1} D_{x_{i} x_{j}} b_{\lambda}^{i j}(x, t) g(x, t)\right)\right|_{t \rightarrow t-\lambda \tau} h(x, t)\right\} d x d t \\
-\int_{t_{0}}^{t_{1}} \int_{\Omega} \sum_{\lambda=-1}^{1}\left\{a_{\lambda}(x, t) g_{t}(x, t+\lambda \tau)-b_{\lambda}^{i j}(x, t) g_{x_{i} x_{j}}(x, t+\lambda \tau)\right\} \\
\cdot h(x, t) d x d t=0, \quad \forall u \in D\left(N_{1}\right), \forall g, h \in D\left(N_{1 u}^{\prime}\right) .
\end{gathered}
$$

From that, we come to the following:

$$
\begin{array}{r}
-\left.\sum_{\lambda=-1}^{1}\left(D_{t}\left(a_{\curlywedge} g(x, t)\right)-D_{x_{i} x_{j}}\left(b_{\lambda}^{i j} g(x, t)\right)\right)\right|_{t \rightarrow t-\lambda \tau}=\sum_{\lambda=-1}^{1}\left\{a_{\lambda} g_{t}(x, t+\lambda \tau)-b_{\lambda}^{i j} g_{x_{i} x_{j}}(x, t+\lambda \tau)\right\}, \\
\forall g \in D\left(N_{1 u}^{\prime}\right) .
\end{array}
$$

That is true if and only if

$$
\begin{gathered}
-a_{-\lambda}(x)=a_{\lambda}(x), \quad \lambda=1,-1, \quad a_{0}(x)=0,\left.\quad b_{\lambda}^{i j}(t)\right|_{t \rightarrow t-\lambda \tau}=b_{-\lambda}^{i j}(t), \quad \lambda=-1,0,1, \\
i, j=\overline{1, n}, \quad \forall(x, t) \in Q .
\end{gathered}
$$

Under the fulfilment of that conditions, the corresponding functional is given by

$$
\begin{array}{r}
F_{N_{1}}[u]=\frac{1}{2} \int_{t_{0}}^{t_{1}} \int_{\Omega}\left\{a_{1}(x) u_{t}(x, t-\tau) u(x, t)-a_{1}(x) u_{t}(x, t+\tau) u(x, t)+b_{1}^{i j}(t-\tau) u_{x_{i}}(x, t-\tau)\right. \\
\left.\cdot u_{x_{j}}(x, t)+b_{0}^{i j}(t) u_{x_{i}}(x, t) u_{x_{j}}(x, t)+b_{-1}^{i j}(t+\tau) u_{x_{i}}(x, t+\tau) u_{x_{j}}(x, t)\right\} d x d t+\text { const. }
\end{array}
$$

Let us consider an example when this criterion of potentiality fails.

Example 4.2. Consider the equation:

$$
\begin{aligned}
N_{2}(u)= & u_{t}(t, x)-2 u(t, x+2 \tau) u_{x}(t, x+2 \tau)-2 u_{x}(t, x) u(t, x-2 \tau)-2 u(t, x) u_{x}(t, x-2 \tau) \\
& +\frac{1}{2} u_{x x x}(t, x+2 \tau)+\frac{1}{2} u_{x x x}(t, x-2 \tau)=0, \quad(t, x) \in Q_{T}=(0, T) \times(-\infty,+\infty) .
\end{aligned}
$$

Let us note that this equation is a Korteweg-de Vries' equation $\tau=0$. 
We denote

$D\left(N_{2}\right)=\left\{u \in U: C_{t, x}^{1,3}((0, T) \times(-\infty,+\infty)):\left.u\right|_{t=0}=u_{0}(x),\left.u\right|_{t=T}=u_{1}(x), \lim _{|x| \rightarrow+\infty} D_{x}^{n} u=0,(n=\overline{0,3})\right\}$.

It is easy to be convinced that operator (4.8) is not potential on set (4.9) relative to the bilinear form:

$$
\Phi(v, g)=\int_{0}^{T} \int_{-\infty}^{+\infty} v(t, x) \cdot g(t, x) d x d t, \quad v \in V, g \in U
$$

Here, $V=\left\{v \in C_{t, x}^{0,3}((0, T) \times(-\infty,+\infty)): \lim _{|x| \rightarrow+\infty} D_{x}^{n} v=0(n=\overline{0,3})\right\}$.

We define the integrating operator $M$ as $M v(t, x)=\int_{-\infty}^{x} v(t, y) d y$.

Then, the operator,

$$
\begin{gathered}
\widetilde{N}(u) \equiv M N_{2}(u)=\int_{-\infty}^{x} u_{t}(t, y) d y-u^{2}(t, x+2 \tau)-2 u(t, x) u(t, x-2 \tau) \\
+\frac{1}{2} u_{x x}(t, x+2 \tau)+\frac{1}{2} u_{x x}(t, x-2 \tau) d y
\end{gathered}
$$

is potential on set (4.9) relative to bilinear form (4.10). The corresponding functional $F_{N_{2}}[u]$ has the form:

$$
\begin{aligned}
& F_{N_{2}}[u] \\
& \quad=\frac{1}{2} \int_{0}^{T} \int_{-\infty}^{+\infty}\left\{u(t, x) \int_{-\infty}^{x} u_{t}(t, y) d y-u_{x}(t, x-\tau) u_{x}(t, x+\tau)-2 u^{2}(t, x+\tau) u(t, x-\tau) d y\right\} d x d t .
\end{aligned}
$$

Indeed, using (4.12), we find that

$$
\begin{gathered}
\delta F_{N_{2}}[u, h]=\int_{0}^{T} \int_{-\infty}^{+\infty}\left\{\int_{-\infty}^{x} u_{t}(t, y) d y+\frac{1}{2} u_{x x}(t, x+2 \tau)+\frac{1}{2} u_{x x}(t, x-2 \tau)-2 u(t, x) u(t, x-2 \tau)\right. \\
\left.-u^{2}(t, x+2 \tau) d y\right\} h(t, x) d x d t, \quad \forall u \in D(N), \forall h \in D\left(N_{u}^{\prime}\right) .
\end{gathered}
$$

From the condition $\delta F_{N}[u, h]=0, u \in D(N)$, for all $h \in D\left(N_{u}^{\prime}\right)$, we obtain

$$
\begin{gathered}
\widetilde{N}(u) \equiv M N_{2}(u) \equiv \int_{-\infty}^{x} u_{t}(t, y) d y-u^{2}(t, x+2 \tau)-2 u(t, x) u(t, x-2 \tau)+\frac{1}{2} u_{x x}(t, x+2 \tau) \\
+\frac{1}{2} u_{x x}(t, x-2 \tau) d y=0, \quad u \in D(N),
\end{gathered}
$$


This equation is equivalent to (4.8).

Let us note that the formula $I[u]=\int_{-\infty}^{+\infty}(1 / 2) u_{x}(t, x-\tau) u_{x}(t, x+\tau)+u^{2}(t, x+\tau) u(t, x-$ $\tau) d x$ defines the first integral $I[u]=$ const of $(4.8)$.

\section{Acknowledgment}

The work of V. M. Sovchin was partially supported by the Russian Foundation for Basic Research (Projects 09-01-00093 and 09-01-00586).

\section{References}

[1] V. M. Filippov, V. M. Savchin, and S. G. Shorokhov, "Variational principles for nonpotential operators," Journal of Mathematical Sciences, vol. 68, no. 3, pp. 275-398, 1994.

[2] I. A. Kolesnikova, A. M. Popov, and V. M. Savchin, "On variational formulations for functional differential equations," Journal of Function Spaces and Applications, vol. 5, no. 1, pp. 89-101, 2007.

[3] V. M. Savchin, "Helmholtz's conditions of potentiality for PDE with deviating arguments," in Proceedings of the 35th Scientific Conference of the Departement of Physico-Mathematical and Natural Science, Part 2. Mathematical Sections, p. 25, M.: RUDN, May 1994.

[4] A. M. Popov, "Potentiality conditions for differential-difference equations," Differentsial'nye Uravneniya, vol. 34, no. 3, pp. 422-424, 1998.

[5] V. M. Savchin and S. A. Budochkina, "On the structure of a variational equation of evolution type with a second derivative with respect to $t$-derivative," Differentsial'nye Uravneniya, vol. 39, no. 1, pp. 118-124, 2003.

[6] V. M. Savchin, "An operator approach to Birkhoff's equation," Vestnik RUDN, vol. 2, no. 2, pp. 111-123, 1995.

[7] L. E. El'sgol'tz, Qualitative Methods in Mathematical Analysis, vol. 1 of Translations of Mathematical Monographs, American Mathematical Society, 1964.

[8] A. L. Skubachevskii, Elliptic Functional-Differential Equations and Applications, Birkhäuser, Basel, Switzerland, 1997.

[9] G. A. Kamenskiı̌, "Variational and boundary value problems with deviating argument," Differentsial'nye Uravneniya, vol. 69, no. 8, pp. 1349-1358, 1970. 


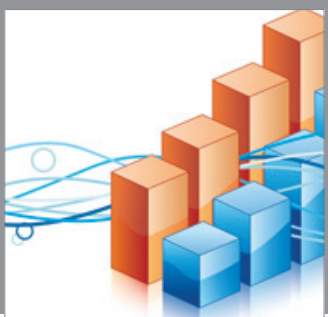

Advances in

Operations Research

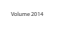

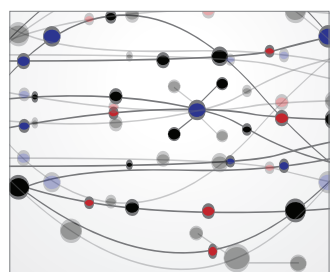

\section{The Scientific} World Journal
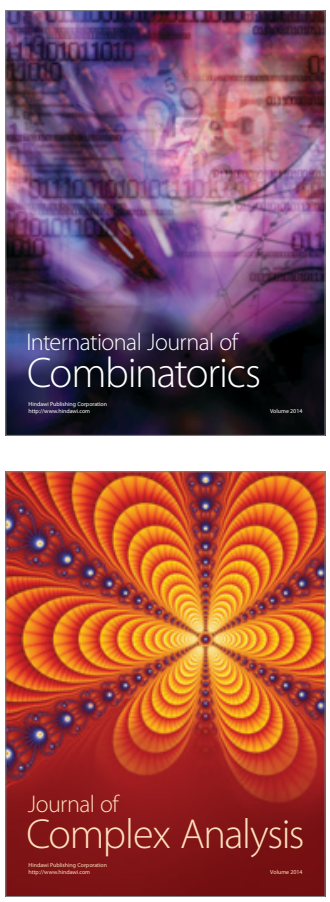

International Journal of

Mathematics and

Mathematical

Sciences
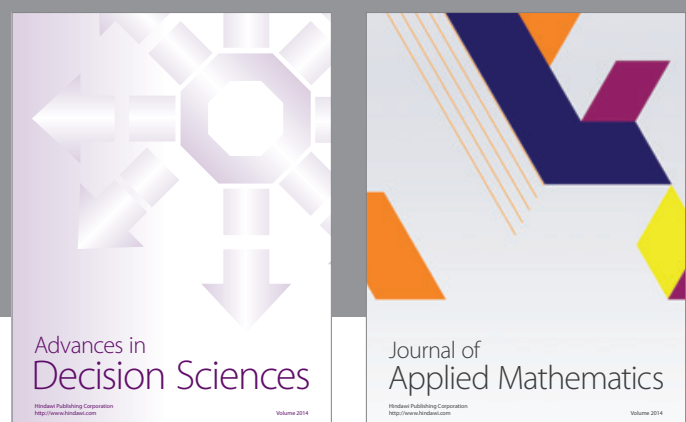

Journal of

Applied Mathematics
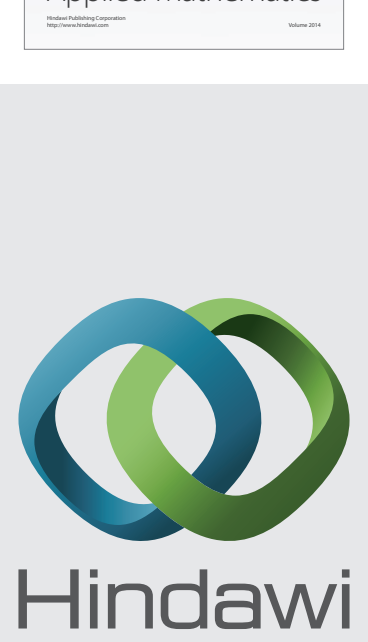

Submit your manuscripts at http://www.hindawi.com
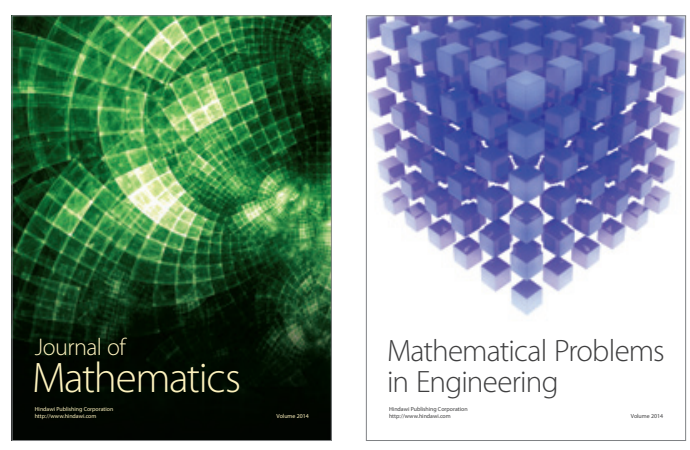

Mathematical Problems in Engineering
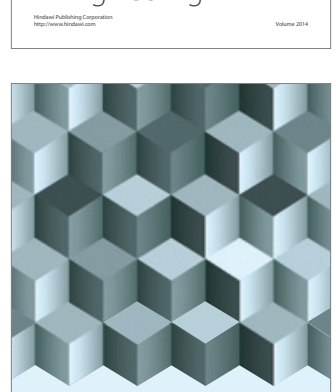

Journal of

Function Spaces
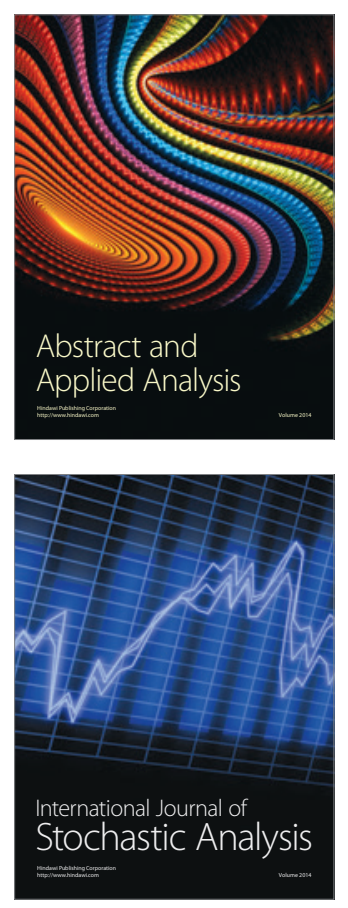

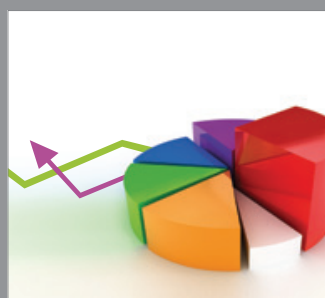

ournal of

Probability and Statistics

Promensencen
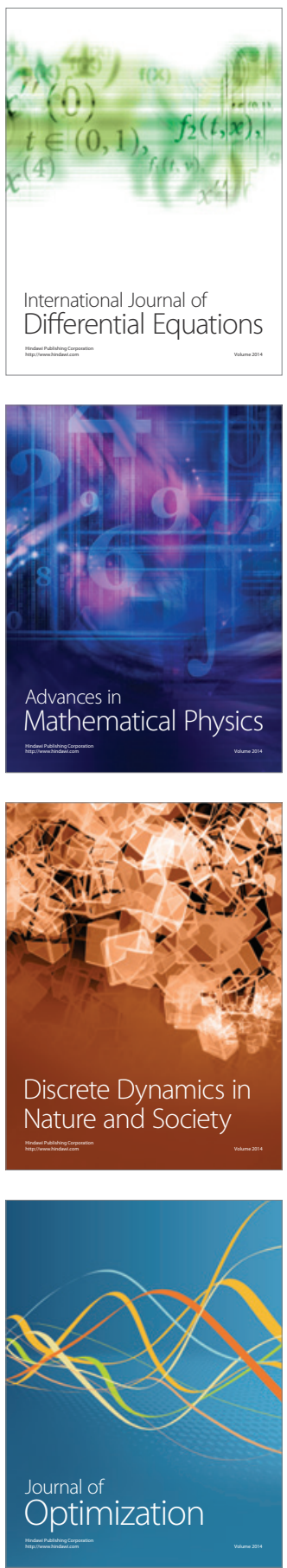